\title{
Microarrays in Cancer: Research and Applications
}

\author{
Michael F. Ochs ${ }^{1}$ and Andrew K. Godwin ${ }^{2}$ \\ ${ }^{1}$ Bioinformatics, Department of Information Science and Technology and ${ }^{2}$ Department of Medical Oncology, \\ Fox Chase Cancer Center, Philadelphia, PA, USA
}

BioTechniques 34:S4-S15 (March 2003)

\section{INTRODUCTION}

Cancer is an important public health concern in the United States and around the world. After heart disease, it is the second leading cause of death, accounting for $23.0 \%$ of deaths in the U.S. (1). In the year 2003, it is estimated that about 1334100 new cases of invasive cancer will be diagnosed in the U.S. (2) and that this number will double by the year 2050 (3). In addition, over one million cases of basal and squamous cell skin cancer will be diagnosed annually $(1,2)$. It is estimated that 556500 Americans will die from cancer in 2003, corresponding to 1500 deaths per day. Among men, the most common cancers are cancers of the prostate (33\%), lung and bronchus (14\%), and colon and rectum (11\%) (2). Among women, the three most commonly diagnosed cancers are cancers of the breast (32\%), lung and bronchus (12\%), and colon and rectum (11\%) (2). In 1987 , lung cancer surpassed breast cancer as the leading cause of cancer death in women and is expected to account for at least $25 \%$ of all female cancer deaths in the coming years. The good news is that following more than 70 years of increases, the recorded number of total cancer deaths among men and women in the U.S. has declined for the first time. For all cancer types and races combined, cancer death rates declined by $1.5 \%$ per year in males and by $0.6 \%$ per year in females from 1992 to 1999. These somewhat encouraging trends are primarily associated with improved screening techniques and the subsequent increase in diagnosis at an early stage when most, but not all cancers are more successfully treated. Unfortunately, most current cancer therapies have limited efficacy in curing late-stage disease. Therefore, there continues to be a need to develop new approaches to $(i)$ diagnose cancer early in its clinical course, (ii) more effectively treat advanced stage disease, (iii) better predict a tumor's response to therapy prior to the actual treatment, and (iv) ultimately prevent disease from arising through chemopreventive strategies. These goals can only be accomplished through a better understanding of how certain genes and their encoded proteins contribute to disease onset and tumor progression and how they influence the response of patients to drug therapies. Innovations in genetic, biological, and biochemical approaches are necessary to realize these goals.

In this aspect, we are in an era of unprecedented opportunity for addressing these questions and uncovering the molecular basis of cancer. It is now clear that new approaches to science, based on comprehensive molecular analysis, offer remarkable new opportunities to enhance the framework of our knowledge of cancer. It has been evident from the beginning of the molecular study of cancer that this is not one disease but many, and that any particular cancer arises as the result of the gradual accumulation of genetic changes within a single cell. New technologies offer the potential to describe specific types of genetic changes and the patterns of altered gene product expression and function that define the actual definition of any cancer in the context of, but not strictly dependent upon, its site of origin. We anticipate that these new technologies will lead to the identification of previously undetectable features of the molecular basis of individual tumor characteristics and profile progression and response to therapy. What is particularly evident is the need to link the information gained from these new technologies with a wealth of clinical information (obtained from pathologists to oncologists) associated with each clinical sample. DNA microarrays are one of these new powerful technologies. In the 8 years since its inception, microarray technology has become a major tool for the investigation of global gene expression of all aspects of human disease and in biomedical research. The range of applications of microarray technology is enormous. Recent studies in human cancer have demonstrated that microarrays can be used to develop a new taxonomy of cancer, including major insights into the genesis, progression, prognosis, and response to therapy on the basis of gene expression profiles. We are just beginning to be able to answer clinically important questions about cancer, such as which tumors will behave aggressively, which will remain indolent, and which are likely to respond to different therapies. Furthermore, the list of potential uses of this technique is not limited to cancer research as evident by the exponentially growing number of manuscripts that have recently been published using these methods (Figure 1).

\section{CANCER AND LARGE-SCALE GENE EXPRESSION CHANGES}

The concept of "discovery science" is best illustrated by the human genome project $(4,5)$. This type of approach involves the identification of the components of a system without the prior formulation of hypotheses as to how these components function (6). This scientific method has spawned many global genomic 
approaches to begin characterizing a vast number of biological systems. Traditionally, studies measuring the differences between cell types or cell pathways following perturbation of the cell have been carried out at the level of transcribed mRNA using methods such as differential display (DD) $(7,8)$, representational difference analysis (RDA) (9), and suppressive subtractive hybridization (SSH) $(10,11)$. However, these methods, although fruitful and still in use, have limited scope in terms of the number of gene expression patterns that can be analyzed. Therefore, more robust methods, including serial analysis of gene expression (SAGE) (12), massive parallel signature sequencing (MPSS) (13), and cDNA arrays and chip technologies (14-18) have taken their places.

\section{Development of Microarrays}

DNA microarrays are rapidly becoming a fundamental tool in genomic research. Microarray methods were initially developed to study differential gene expression using complex populations of RNA (14-18). Refinement of these methods now permits the analysis of copy number imbalances and gene amplification of DNA (19) and the detection of small deletions or insertions in tumor suppressor genes (20), and they have recently been applied to the systematic analysis of expression at the protein level (21). For the purpose of this review we will focus on spotted microarrays.

Spotted arrays. The term "spotted microarray" refers to a 2dimensional series of elements on a surface, where each element

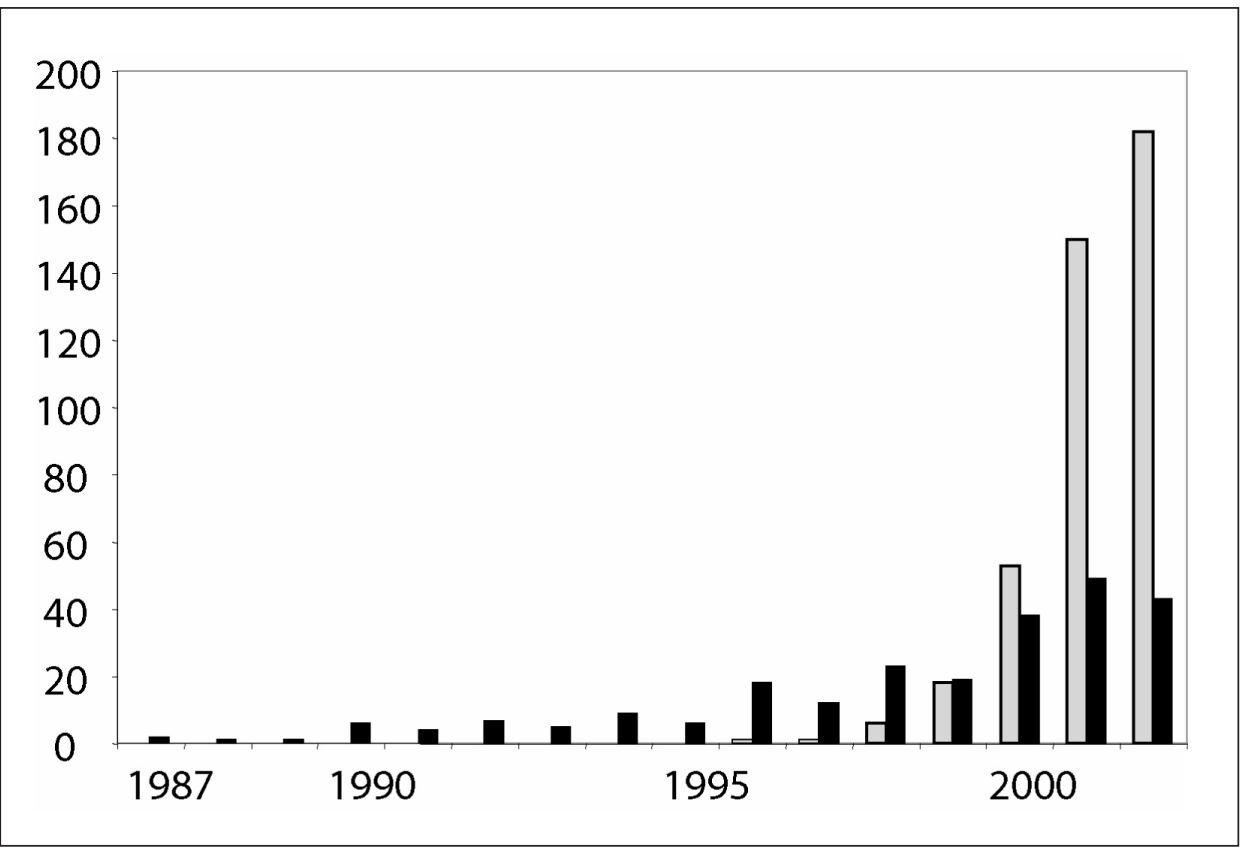

Figure 1. The growth in microarray publications in cancer research. The rapidly increasing number of publication describing DNA microarray analysis of cancers indicates the growing interest in this technique for cancer researchers (gray bars). Subtractive hybridization is another technique designed to isolate differentially regulated gene transcripts. By comparison with cancer microarray papers, the more labor-intensive subtractive hybridization method has generated fewer manuscripts over a much longer time period (black bars). Searches were performed at http://www.ncbi.nlm.nih.gov/entrez/query.fcgi? db=PubMed using the search strings "cancer and DNA and microarray" (gray bars) and "subtractive hybridization and cancer" (black bars). (Figure kindly supplied by Dr. Geraldine O'Neill of The Children's Hospital Westmead, Westmead, NSW, Australia.) of the array is an aliquot of some nucleic acid that has been deposited in its specific location [as opposed to being synthesized at that location, base by base in situ (22)]. Spotted arrays are manufactured using $\mathrm{x}-\mathrm{y}$-z robots that use hollow pins to deposit cDNAs (PCR products), clones, or short and long oligonucleotides onto specially coated glass microscope slides (18). Spot sizes range between 50 and $500 \mu \mathrm{m}$ in diameter (depending on the type of pin used, e.g., pin and ring versus quill or slit pin) (23), and arrays that contain up to 80000 spots can be obtained, however, lower density arrays are more common (from 100 elements per $\mathrm{cm}^{2}$ to as many as 25000 elements per $\left.\mathrm{cm}^{2}\right)$. Gene sequences to be arrayed are selected from several public databases, which contain resources to access well-characterized genes and expressed sequence tags (ESTs) representative of genes of unknown function. The clones chosen are amplified from appropriate cDNA libraries by PCR and purified before spotting on the solid support.

The strength of this technology is its flexibility: enabling relatively rapid iteration of experiments, where the number of array elements is in the range of hundreds to low thousands, and where 20 to 100 custom designed arrays can be fabricated for a particular experiment. In addition to their lower price and this flexibility in design, spotted arrays offer the ability to interrogate many surface-bound probes simultaneously with a very small amount of labeled target sample applied in a small volume (in contrast to the use of large filters as substrates for hybridization that require labeled probes applied in much larger volumes). Simultaneous expression analysis of two biological samples, such as untreated cells compared with treated cells or healthy tissue compared with cancer, also offers an enormous advantage for any pairwise analysis. Another strength of the technology is in enabling the study of genes that have not yet been sequenced (i.e., spotting ESTs offers the potential for the discovery of new genes and defining their role in disease). The major disadvantage of spotted arrays, which is due to the variability in spot quality from slide to slide, is that they provide information only on the relative gene expression between specific cells or tissue samples as opposed to direct quantification of RNA expression.

Affymetrix GeneChips ${ }^{\mathrm{TM}}$. A competing microarray technology that has garnered much attention is the GeneChip (Affymetrix, Santa Clara, CA, USA). High-density oligonucleotide GeneChips are produced by synthesizing tens of thousands of short oligonucleotides in situ onto glass wafers, one nucleotide at a time, using a combination of photolithography and lightdirected solid-phase DNA synthesis $(16,22)$. Generally, GeneChips are 
designed with 16 to 20 , preferably nonoverlapping 25-mers representing each gene on the array. Each oligonucleotide on the chip is matched with an almost identical one, differing only by a central, single-base mismatch. This mismatched oligonucleotide serves as an internal control for hybridization specificity and allows for determination of the degree of nonspecific binding by comparison of target binding intensity between the two partner oligonucleotides. GeneChip oligonucleotide probe arrays are ideally suited for applications involving analysis of large amounts of known sequence content (e.g., whole genome expression monitoring, where the vast majority of genes for a given organism have already been sequenced and can be synthesized as probes), where high quality is required for every array utilized, and where the same array design can be used repeatedly. Another advantage of Affymetrix GeneChips is their ability to measure the absolute expression of genes in cells or tissues. The sensitivity of the GeneChip enables the detection of mRNAs present at levels as low as 1 transcript in 100000 ; at the extreme, sensitivity has been reported as low as 1:1000 000 (16). Their disadvantages, in addition to their higher costs, include the inability to simultaneously compare, on the same array, the degree of expression of two related biological samples. In addition, oligonucleotide-based microarrays require a priori knowledge of the gene sequences and complex computational manipulation to convert the 40 feature signals into an actual expression value. More recently, oligonucleotide arrays have been developed that combine some of the flexibilities and qualitative advantages associated with the use of synthetic probe arrays with the benefits of simultaneous analysis afforded by spotted glass arrays (24).

\section{Global Gene Expression in Tumor Biology}

Identification of tumors by histology or immunohistochemistry yields information regarding tumor origin, tumor type, and tumor stage or grade, which to date still provide the most relevant prognostic information. However, staining patterns that are microscopically observed cannot predict the underlying biochemical and genetic events that are important for clinical outcome. Multiple genetic changes that result in the activation of oncogenes, increased production of growth factors, loss of growth inhibitory cytokines, or loss of function of tumor suppressor genes result in an imbalance of growth and survival regulation. The result of such derangements is a persistent pathologic communication state between the tumor cell and the host in which tumor cells successfully invade and metastasize because of somatic genetic progression that supports invasion and survival. While cancer researchers have made great strides in uncovering parallel molecular mechanisms that are involved between many different cancer types, the set of genes required for successful tumorigenesis is still debated. Clinically, it can be observed, for example, that two patients harboring similar tumors according to their cellular morphology may have two different clinical outcomes and/or may respond differently to the same treatment. Therefore, cancer researchers are applying cDNA and oligonucleotide microarrays to study several thousand genes at once to enhance our current tumor classification system (25-27), while concomitantly providing insights into pathogenesis, diagnosis, prognosis, therapeutic targets, and clinical outcome of tumors $(16,26,28-31)$.
Tumor classification. In a landmark study, Golub and colleagues were among the first to correlate gene expression profiles with tumor classification (32). They were able to distinguish acute myeloid leukemia (AML) from acute lymphoblastic leukemia (ALL) based on gene expression alone in 36 out of 38 bone marrow samples. Yeoh and colleagues extended these types of studies and have demonstrated in pediatric ALL that expression profiling could not only accurately identify the known prognostically important leukemia subtypes, but could further enhance the ability to assess a patient's risk of failing therapy (33). Other groups have also used gene expression pattern analysis to classify, at the molecular level, ovarian tumors $(34,35)$, breast tumors (36,37), B-cell lymphoma (38), cutaneous melanoma (28), and lung adenocarcinoma $(39,40)$. Likewise, in a recent study analyzing molecular profiles of nonneoplastic and neoplastic prostate samples, Dhanassekaran and colleagues established a signature expression profile of healthy prostate, benign prostatic neoplasia, localized prostate cancer, and metastatic prostate cancer (41). These studies have demonstrated the feasibility of combining large-scale molecular analysis of expression profiles with classic morphologic and clinical methods of staging and grading cancer for better diagnosis and outcome prediction.

Tumor-specific molecular markers. As noted above, tumors can be divided clinically into those that originate in solid organs such as the kidney, bowel, or lung, and those that originate in the hematopoietic system. In fact, this distinction is helpful in considering how to apply array technology to expression monitoring of cancer, because issues of sample preparation and tissue heterogeneity are of particular concern in performing and interpreting experiments with solid tumors. Nevertheless, there is widespread optimism in the scientific community that microarray-based expression monitoring will help decipher the information hidden in sequenced genomes. There is a growing number of research groups that have focused on identifying subsets of genes that show differential expression between healthy tissues or cell lines and their tumor counterparts to identify biomarkers for several solid tumors, including ovarian cancer (42-48), oral cancer (49,50), endometrial cancer (51), melanoma (52), and colorectal cancer $(53,54)$. Unfortunately, the realization from most of these studies is that the generation of data is not enough; one must be able to extract from it meaningful information about the system being studied. Furthermore, the issue of tissue homogeneity needs to be carefully considered. Global gene expression surveys assume that information of interacting cell populations can be globally investigated by analyzing gene expression patterns from heterogeneous tissue samples. Total RNA from tissue fragments (i.e., high tumor content versus low tumor content) is isolated, labeled, probed on an array, and the status of gene expression quantified. However, it is not hard to imagine that genes from cell subpopulations are also included and thus may contaminate the results. Therefore, much effort has been directed towards using microdissected cell populations, which in turn have their own advantages and disadvantages (55). Nevertheless, as more and more researchers rush to embrace the new techniques, one of the most important challenges facing these investigators will be determining which of the plethora of new differentially expressed genes is biologically relevant to the tumor system being studied. Even when rigorous efforts are made to minimize the number of variables in a mi- 
croarray study, there may be an unmanageable number of differentially expressed genes that will contribute excessive background values. Therefore, the greatest contributions to understanding function will likely come from directly combining microarray data with other sources of genomic and biomedical information. Algorithms for integrating different types of data are already showing promise and integrating clinical data from patients' records has been proposed. By combining expression microarray analysis with other approaches, such as cytogenetic techniques, it may be possible to focus on a significantly smaller subset of genes of direct relevance to tumor biology (56). In practice, recent studies have combined expression arrays and comparative genomic hybridization array techniques on breast cancer cell lines and identified a limited number of genes that were both amplified and overexpressed $(57,58)$. Efforts have also been initiated to apply in concert DNA microarrays and proteomics approaches to better understand the regulatory events involved in normal and disease processes (38). This is important given that the measurement of transcribed mRNA alone is not sufficient for the characterization of biological systems. It is clear that continued advancements in the comprehensive analysis of protein products in conjunction with the already mature methods in global measurement of mRNA expression will eventually help to uncover the molecular basis of cancer.

\section{METHODS FOR ANALYZING MICROARRAY DATA}

The fundamental change typifying cancer is the clonal proliferation of a single cell, which requires activation of the cell cycle and the overriding of cellular programs (e.g., apoptosis) that guard against proliferation. A number of specific cellular malfunctions have been identified, including loss of function of key tumor suppressor genes such as p53 (59-61), gain of function mutations of cell growth regulators such as c-KIT $(62,63)$, and activation of proto-oncogenes such as c-MYC (64). Since cells within complex organisms have built-in safeguards against proliferation, a single adverse event in the cell is generally not adequate to initiate cancer (65), but two or more events are required to take a cell from normal to malignant, often including a definite sequence of cellular events (66). During these changes the expression levels of numerous genes are expected to undergo changes as the cell adjusts itself to a new program. These changes take place within a background of numerous routine expression changes or even stochastically varying levels of gene expression. The issue for microarray measurements then becomes identifying changes related to the process of interest, e.g., tumorigenesis, in a background of routine differences in expression between individual sets of cells. Such sets of cells may be measurements from different patients, different times in the 
same individual, or different tissue types within an individual.

The multitude of possible cellular events that can give rise to cancer lead to a diversity in types of cancer not seen in other diseases. Often cancers that appear histologically similar can have dramatically different responses to standard therapies and different courses of development (67). Since these differences in behavior are likely to be reflected in differences in the set of genes expressed, one promising use for microarrays is to more finely differentiate cancers using gene expression levels to bolster standard histology. The goal is to allow therapies, including the level of aggressiveness of treatment, to more closely match the underlying disease, hopefully reducing side effects in cases of low risk and increasing cure rates in cases of high risk. In this case the goal is identification of an expression profile that can be used to type the cancer.

\section{Methods of Analysis for Classification}

To use microarray measurements to distinguish cancer types, it is necessary to identify patterns for each subtype that uniquely distinguish that subtype from all others. This requires some form of pattern recognition, with the methods used generally borrowed from the fields of statistics, computer science, and data mining. Since the data generated in a microarray experiment usually comprise a matrix of measurements (Figure 2), methods of analysis developed for matrix manipulations can be applied to microarray measurements as well. Generally the individual rows of the matrix identify different genes or nucleotide sequences with the individual columns identifying different conditions. For the purposes of cancer identification, the columns would represent different tumors, patients, or tissues. The goal for an analysis aimed at identifying tumor profiles would be to divide the conditions into sets linked to tumor or tissue type based on expression levels of the genes. Two general types of methods can be used. The first type is unsupervised learning, in which there is no a priori identification of the group in which a condition (e.g., sample) should be placed (i.e., the type of tumor). The second type is supervised learning in which a well-defined learning set exists (e.g., each sample identified as to type of tumor) to allow an algorithm to develop a test that will be applied to additional samples of unknown type.

Unsupervised learning includes a set of methods that attempt to define groups within the data, often called clusters. These clusters are then interpreted in light of other information. For instance, in a study of gene expression in the NCI60 cell lines, the clusters identified could be linked to the tissue of origin of the tumor (68). The algorithms for clustering include a number of different methods, such as hierarchical and K-means clustering (69-71), bootstrapping or jackknife methods (72,73), methods which test for poor data (74), clustering based on statistical methods such as singular value decomposition or principal component analysis (75), fuzzy clustering methods (76,77), and self-organizing maps (78). Each of these methods has its own advantages and limitations. While there has been success in the use of these algorithms, no clear theoretical advantage exists for any given algorithm in application to expression data, so the safest approach is to apply multiple algorithms and to try to determine which functions best on the specific data set.

Supervised methods are generally borrowed from the field of computer science and machine learning. These methods require a training set where the identification of each condition has been made by other means. For example, in their analysis, J. Khan et al. first trained the algorithm using a subset of the full data where the identification of the malignancy had already been made (79). The supervised learning methods that have been applied to gene expression data include support vector machines (SVM) (80), multidimensional scaling (MDS) (26), and artificial neural networks (79). The main goal behind these algorithms is to define a set of criteria that allow the maximal separation of the conditions into the correct types.

\section{Specifics of Algorithms for Unsupervised Learning}

Because of its ready availability due to the release of an easily used version by Eisen and colleagues (69), the pattern recognition algorithm most widely applied to microarray data is hierarchical clustering. Hierarchical clustering measures similarity between the genes based on expression profiles across conditions. This measurement requires a definition of similarity that is provided by a metric. The most common metric is Euclidean distance, which measures how different two profiles are taking into account the magnitude of signals (i.e., the vector $[10,20,30]$ is very distant from the vector $[1,2,3])$. Another common distance metric is Pearson correlation coefficient, which measures the shape or direction (i.e., the vector $[10,20,30]$ is at the same point as the vector $[1,2,3])$. The advantage of Pearson correlation as a metric is that genes that vary together but with one

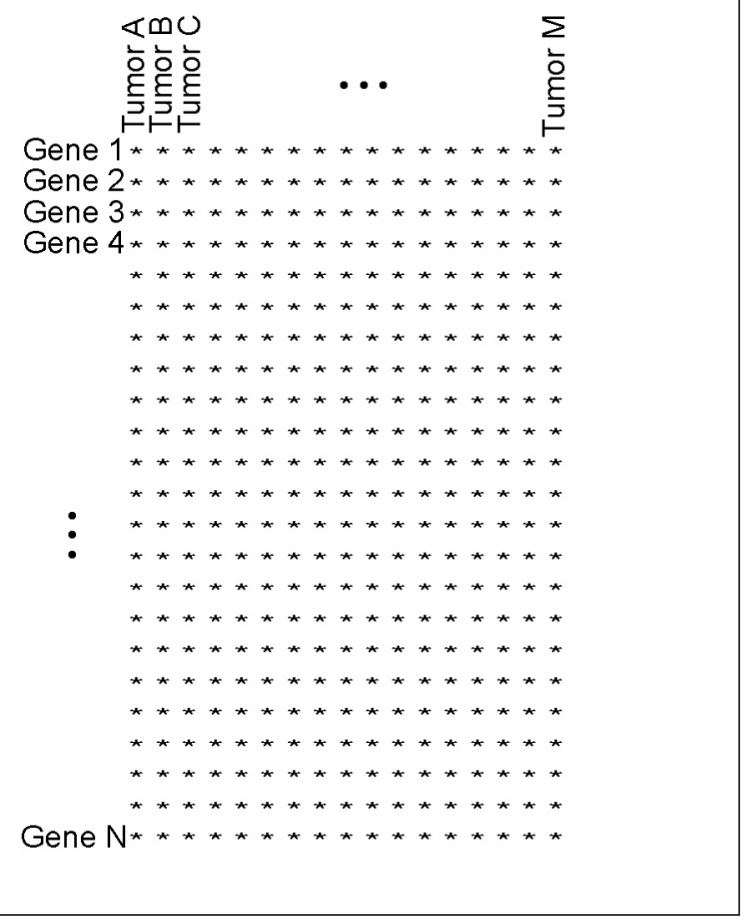

Figure 2. Typical form for microarray data in a cancer-related study following normalization and combining of replicates. The normalized data is a set of values of the expression level for each gene in a number of different conditions, typically in a number of tumors or tissue types. 
much more highly expressed will still cluster together. However, this metric can miss coordinated changes if the changes are comparable in each gene but their background levels of expression vary by different amounts. In addition to the metric, there are multiple methods of building the clusters that vary on use of centroids, nearest members, or farthest members when measuring distances between and among clusters and expression profiles. The details of these issues are beyond the scope of this work, but can be explored in the literature $(81,82)$. Finally, hierarchical clustering generally does not provide clusters but instead provides measures of distance between multiple possible groupings of genes. Generally this is shown as a dendogram and it is left to the user to determine at what level to cut the tree to provide clusters (as shown in Figure 3). This level of control provides power but also makes the method prone to artifactual errors, so that some statistical analysis might be required to determine that the clusters are meaningful.

A second common clustering algorithm borrowed for use with microarray data is $\mathrm{K}$-means clustering $(83,84)$. With this method, the number of clusters must be chosen prior to application of the algorithm. The algorithm then identifies the cluster distribution and assigns members in a way to minimize overall the distances of each point from its cluster. Again a distance metric and method for identifying how to measure the distance of a point relative to the cluster must be chosen.

One problem faced by these clustering methods is the possibility that a single shared bad data point can lead to clustering together two truly unrelated expression patterns. To address this issue, Heyer and colleagues developed a clustering technique that uses jackknife correlation as part of the distance metric (74). In this way, individual data points cannot dominate the underlying clustering.

A further problem with clustering is the issue of the correct number of clusters. In an attempt to answer this problem, Lukashin and Fuchs introduced a method that uses simulated annealing to estimate the number of clusters (71). Simulated annealing is a method borrowed from statistical mechanics that models the slow cooling of a physical system (85), effectively moving from a region where the data plays a minor role in determining the structures of the model to one where the data dominates.

Once the clusters are chosen, there remains the issue of statistical significance. Clearly the wide variety of parameters available in most clustering methods raises the issue of identifying clusters by chance. To validate the results of clustering, Kerr and Churchill introduced a method to use bootstrapping analysis to interpret the results of clustering (72). Bootstrapping estimates the data and noise, which is done here through an analysis of variance (ANOVA) model (86), and clusters models of the data repeatedly. In this way the reliability of the identified clusters can be estimated.

As noted previously, microarray data often take the form of a matrix of measurements, allowing the application of numerous techniques developed for matrix analysis. One such method, sin-

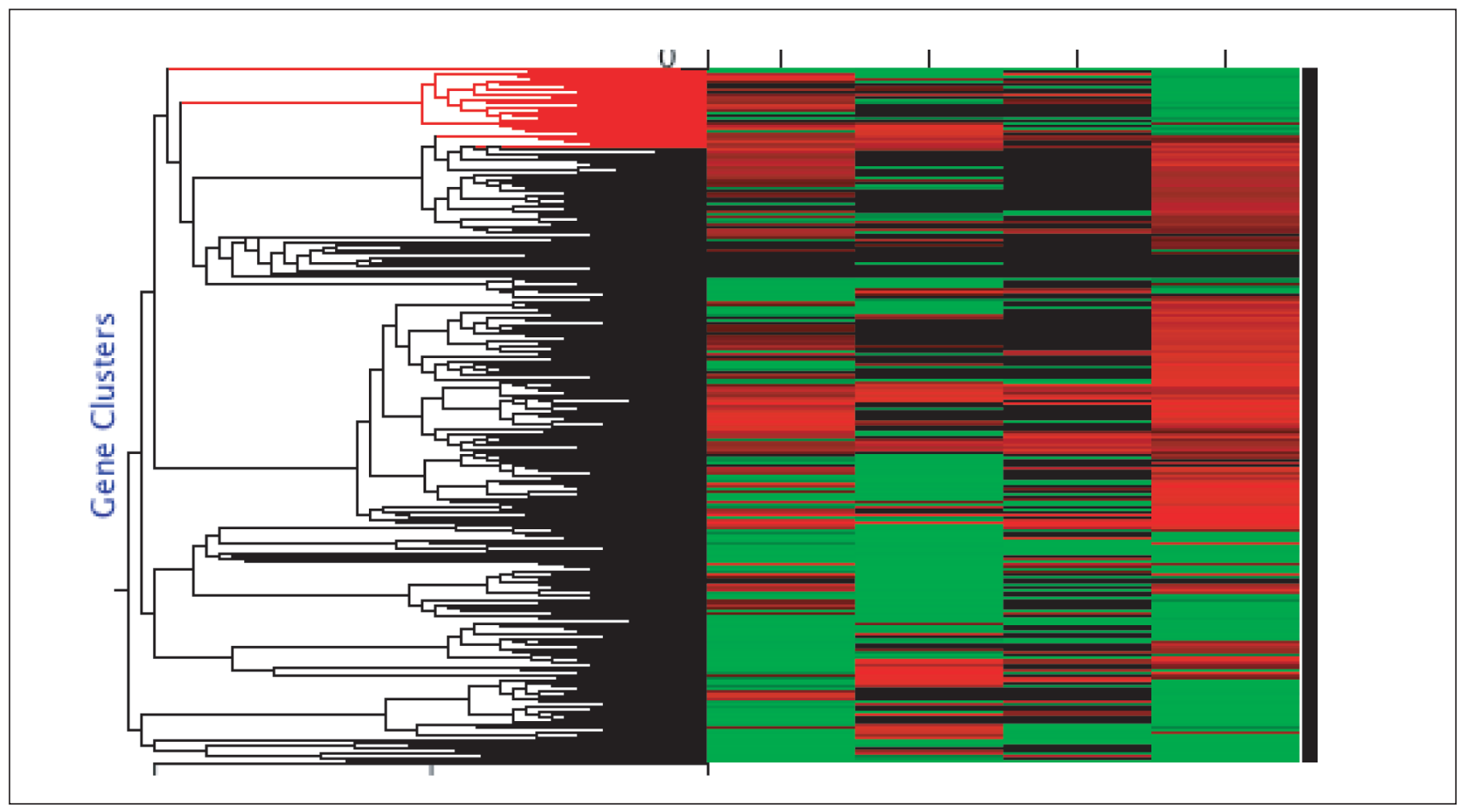

Figure 3. Hierarchical clustering of genes across four conditions. The most widely used pattern recognition method in microarray analysis is hierarchical clustering, in which genes are compared by expression profile across multiple conditions. In this image generated by BioDiscovery's GeneSight ${ }^{\mathrm{TM}}$ (Marina Del Rey, CA, USA), green represents lowered expression relative to the control and red represents higher expression. The dendogram on the left indicates the magnitude of the difference between the expression profiles according to the metric. Clustering requires deciding where to cut the dendogram to define clusters, which can therefore be a few clusters with many genes (cut on far left) or many clusters with few genes (cut on far right). 
gular value decomposition (SVD, or principal component analysis) has been used to create a clustering method as well (75). In SVD, the matrix of measurements is converted into a matrix of principal components and a matrix of scores. The first principal component is usually chosen to maximize the amount of variance within the data explained by that single component, with all other components chosen orthogonal to all proceeding components and in a way to maximize the amount of the remaining variance explained. In this way the data can be reduced in dimensionality, however the number of components to retain is still problematic. The scores then represent how to reconstruct the expression profile of each gene from the principal components, so that plotting the scores for the first three principal components can lead to clustering of the genes into similar groups in three dimensions.

Self-organizing maps are an additional method for clustering (78), which allows the user to guide the analysis by laying out a grid of nodes. These nodes are then moved to match the distribution of the data and each individual data point (i.e., each gene expression profile) is assigned to a node.

All of the methods looked at so far place each gene into a single cluster. This is similar to the logical operation of true (in cluster) or false (not in cluster). However, fuzzy logic systems have been developed that allow intermediate logical assignments between true and false. These methods lead to a clustering method, fuzzy clustering $(76,77)$, that allows genes to be placed into multiple groups with an assignment to the group between 0 (not in group) and 1 (completely within group). This method has the advantage of allowing each gene the possibility of belonging to multiple clusters, which may reflect the underlying biology better (87).

\section{Specifics of Algorithms for Supervised Learning}

One of the most widely used machine learning algorithms is

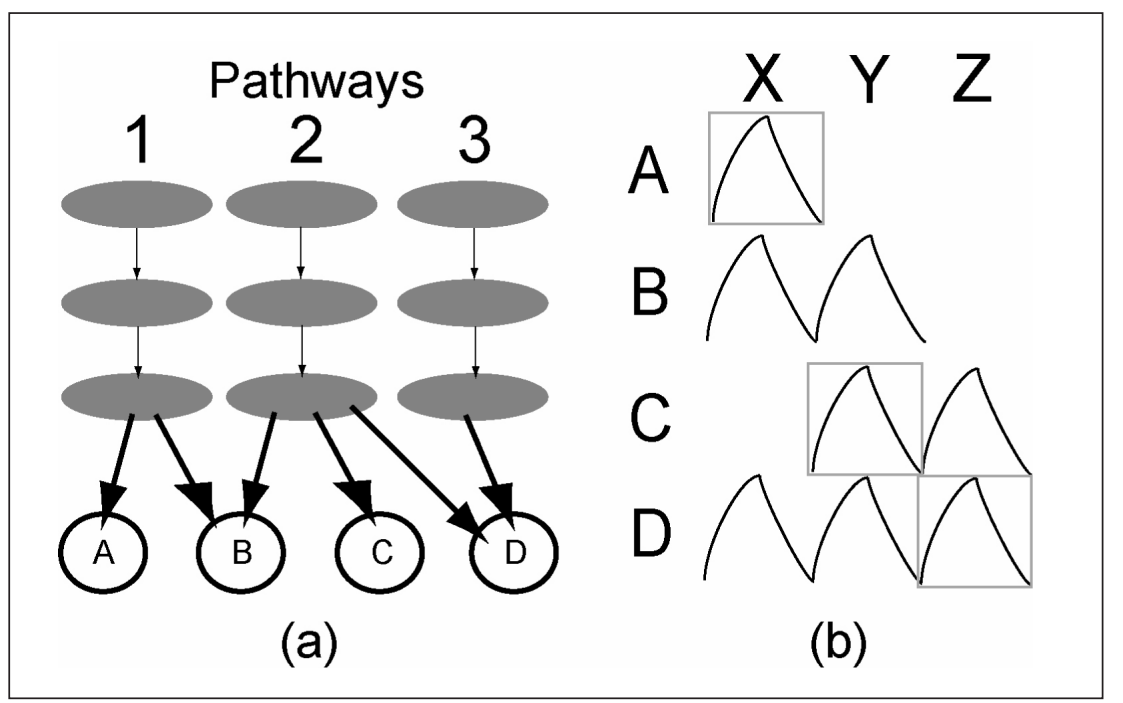

Figure 4. The link from signaling pathways to expression response. This highly simplified diagram demonstrates the problem in using expression analysis for identification of pathway activation even in simple cases (here all cross-talk is pushed to the point of transcription for clarity). The pathways in (a) are transiently active in the order $1 \& 3(\mathrm{X}), 2(\mathrm{Y}), 3(\mathrm{Z})$, resulting in the transcriptional profiles in (b). Algorithms that are unable to assign genes in $\mathrm{B}$ and $\mathrm{C}$ to multiple groups cannot recover the link to the underlying processes or even the correct number of processes. an artificial neural network. These methods were adopted early in the development of microarray analysis in cancer research to identify classification criteria for tumors (79). These algorithms are discussed in this issue by Ringnér and Peterson. Essentially the method involves setting up a network of nodes in layers, with each node fed by all nodes in the previous layer. The strength of the influence of one node on another is tuned by the algorithm to give a final output most matching the predetermined assignment of each sample to a group. Ideally, this results in a nonlinear system tuned to identify the class of a new unknown sample. One drawback to these systems is that it can be hard to interpret the results biologically because of the multiple layers and nonlinearities involved in the neural network.

MDS is a method of mapping complex data into lower dimensional space in a way that maximizes the separation between classes. The classes are predefined (e.g., type of cancer) and the result is a mapping that can be applied to new data to identify the class to which an unknown sample belongs (26).

SVMs are another method of identifying features that separate two predefined classes under study (80). The features are formed by looking at the relationships between each two points in the multidimensional data set. The hyperplane that best separates those feature points is determined and used to classify new samples.

One problem with these methods is that they are trained on a specific set of data measured using a single platform (often within a single laboratory) and including a specific set of genes. For diagnostics to be useful, they must allow for measurements to take place at many locations and ideally allow different platforms for measurement (RT-PCR, Affymetrix GeneChip, Rosetta oligo arrays, etc.). One attempt to allow such broad measurement methods is to create classifiers with the validity of the classification tied to the underlying biology regardless of the measurement method (88). Such a method is described in this issue by Scharpf and colleagues. Essentially to overcome inherent differences in the quantitative reliability of different platforms, a binary classifier is used that measures the probabilities of expression or nonexpression of each gene in each sample based on the data. These are then used in a supervised learning method to identify a subset of genes that together by their presence or absence identify the cancer type.

\section{Methods of Analysis for Exploring Signaling Pathways}

As noted previously, cancer often arises from specific malfunctions in genes involved in cellular signaling. This leads to undesired changes in the activity of specific signaling pathways. Since these pathways generally control gene transcription, these changes are expected to lead to changes in gene expression. The methods discussed so far aim to identify signatures of cancer and type, but do not provide a method of interpreting these patterns in light of signaling pathways. The reason for this is shown diagrammatically in Figure 4. 


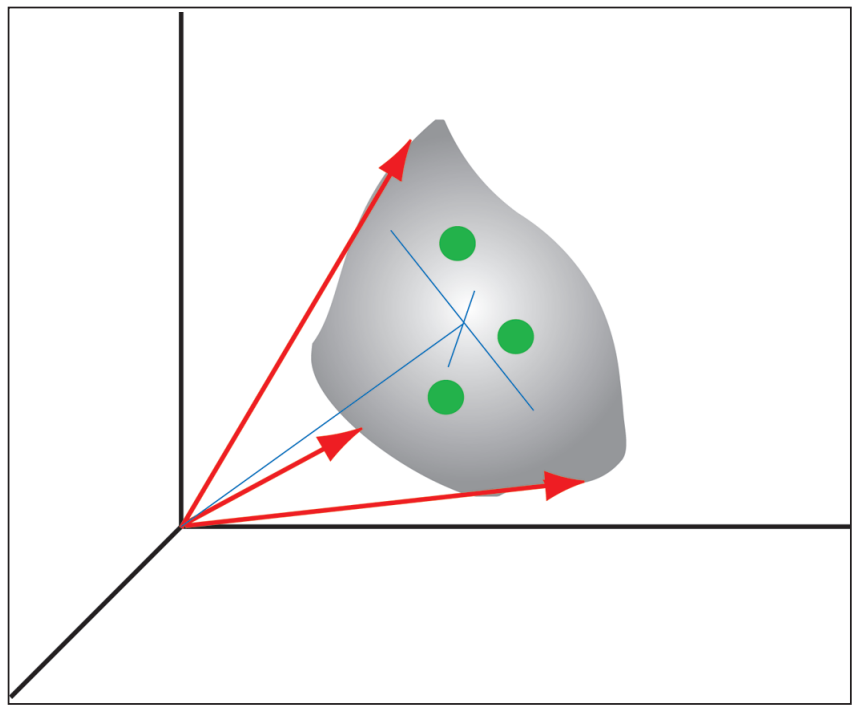

Figure 5. Comparison of methods of identifying patterns in multidimensiona gene expression data. The blue lines represent the PCA solution; the green disks represent fuzzy clusters; and the red vectors represent the desired, physiologically significant patterns spanning the multidimensional data.
Since one result of the activation of a signaling pathway is transcription of a set of genes that generally overlap substantially with genes activated by other pathways, the clustering techniques cannot identify the set related to a specific pathway. This makes it difficult to link expression changes to signaling pathways.

However, pathway information is critical not only to the understanding of cancer development, but also to the design of effective therapeutics in the treatment of cancer. Present cancer treatments, such as radiotherapy and chemotherapy, result in substantial collateral damage to healthy tissues. Targeted therapies would try to alter behavior in a cell-specific manner, affecting only cancer cells. The creation of these therapies will require a detailed understanding of how disrupting specific cellular pathways affects downstream events in cells and an understanding of the signaling and metabolic networks to avoid unintended side effects in treatment (e.g., disrupting a pathway in a healthy cell leading to damage to healthy tissues).

One method of attacking this problem is to model the known pathways and analyze the expression data in light of the existing knowledge of the signaling pathways. The models can be quite detailed, including such information as rate constants, transcriptional initiation and elongation, etc. (89) or highly simplified, merely tracing connections (90). With the provided net- 
work model, the expression data can be used to validate or choose between alternative models (91). The problem that arises with cancer is that the necessary network models remain poorly defined. While there has been some success with limited models $(92,93)$, the gaps in knowledge of signaling networks and potential interactions appear too large to allow widespread use of these models presently (94).

If detailed network models are unavailable, the first step is instead to decompose the observed transcriptional responses into overlapping sets of genes. As can be seen in Figure 4, the desired form for the transcriptional response is generally a minimal structure (as shown in the boxes), since the desired decomposition is into minimal units related to a single pathway or, at least, minimal units related to all synchronized pathways within the data. Mathematically this is equivalent to identifying the minimal edge vectors bounding the data in a multidimensional space (as depicted in Figure 5). Methods that have been proposed to handle the multiple coregulation problem, such as principal component analysis (PCA) and fuzzy clustering (FC), generally operate in the center of the distribution. In addition, the requirement that all principal components (PCs) be orthogonal limits the usefulness of PCA in this type of analysis (see blue lines in Figure 5), since the basis vectors are typically nonorthogonal. For FC, the problem is that the bulk of the data will generally lie on the interior of the distribution rather than the edges, so that the seed clusters will not be located correctly for recovery of the fundamental basis vectors (see green disks in Figure 5).

One approach to recover the nonorthogonal basis vectors (i.e., minimal expression patterns) directly is to use Markov chain Monte Carlo to search through all probable solutions for the one that best reproduces the data. Bayesian Decomposition (87) uses a Bayesian statistical framework (95) that includes a goal of minimizing the structure in the model to identify the minimal patterns and to simultaneously determine the distribution of genes within them. Working on public domain data, it has successfully identified cell cycle regulated genes in the Stanford yeast cell cycle data set $(96,97)$, including multiple coregulation of a number of kinases (87), isolated the transcriptional mating response pattern in Saccharomyces cerevisiae independent of any knowledge of the proteins involved in the signaling pathway (98) in the Rosetta deletion mutant data set (99), and identified patterns and specific genes expressed in mouse organs (100) from the Project Normal data (101). This method is limited since it is driven by the data without a model of the network. As such, it can only recover information with the resolution of the input data - that is, if during the creation of the data three signaling pathways were equally active at all times, those three pathways cannot be identified as separate processes.

\section{CONCLUSION}

In human cancer, the use of microarrays is starting to provide major insights into the genesis, progression, and response to therapy. With the availability of the human genome sequence, the goal of examining the entire transcriptome of normal and malignant cells is now possible. In combination with the continued development of the informatics and data analysis tools necessary to interrogate and interpret these data, this approach promises to revolutionize how we think about cancer and how we are likely to detect and treat cancer in the future. By ultimately combining genomic with proteomic approaches, the information gained from these integrative kinds of studies may lead to the identification of secreted proteins that can be used for sensitive, presymptomatic molecular diagnosis. It may also be possible to find membrane-bound proteins with functions ideally suited for protein therapeutic approaches. In this regard, understanding tumor subclassification is paramount, because expecting cell surface markers that are common to all tumors within a specific malignancy is probably naïve. However, if two or three subclasses of that malignancy can be discerned, it is likely that the strongest predictor genes will qualify as candidates for use in molecular diagnosis. Although there are a variety of technology options available from which to choose, it is clear that the impact of microarray technology on genomic research will be enormous. Going forward, one or another form of this technology will likely be used in all laboratories interested in the study of complex genetic information.

\section{REFERENCES}

1.Greenlee, R.T., T. Murray, S. Bolden, and P.A. Wingo. 2000. Cancer Statistics, 2000. CA Cancer J. Clin. 50:7-33.

2.Jemal, A., T. Murray, A. Samuels, A. Ghafoor, E. Ward, and M.J. Thun. 2003. Cancer Statistics, 2003. CA Cancer J. Clin. 53:5-26.

3.Simmonds, M.A. 2003. Cancer Statistics, 2003: Further decrease in mortality rate, increase in persons living with cancer. CA Cancer J. Clin. 53:4.

4.Lander, E.S., L.M. Linton, B. Birren, C. Nusbaum, M.C. Zody, J. Baldwin, K. Devon, K. Dewar, et al. 2001. Initial sequencing and analysis of the human genome. Nature 409:860-921.

5.Venter, J.C., M.D. Adams, E.W. Myers, P.W. Li, R.J. Mural, G.G. Sutton, H.O. Smith, M. Yandell, et al. 2001. The sequence of the human genome. Science 291:1304-1351.

6.Aebersold, R., L.E. Hood, and J.D. Watts. 2000. Equipping scientists for the new biology. Nat. Biotechnol. 18:359.

7.Liang, P. and A.B. Pardee. 1992. Differential display of eukaryotic messenger RNA by means of the polymerase chain reaction. Science 257:967-971.

8.Martin, K.J. and A.B. Pardee. 1999. Principles of differential display. Methods Enzymol. 303:234-258.

9.Lisitsyn, N. and M. Wigler. 1993. Cloning the differences between two complex genomes. Science 259:946-951.

10.Diatchenko, L., Y.-F. Lau, A. Campbell, A. Chienchik, F. Moqadam, B. Huang, S. Lukyanov, K. Lukyanov, et al. 1996. Suppression subtractive hybridization: A method for generating differentially regulated or tissue-specific cDNA probes and libraries. Proc. Natl. Acad. Sci. USA 93:6025-6030.

11.Wang, X. and G.Z. Feuerstein. 2000. Suppression substraction hybridisation: application in the discovery of novel pharmacological targets. Pharmacogenomics 1:101-108.

12.Velculescu, V.E., L. Zhang, B. Vogelstein, and K.W. Kinzler. 1995. Serial analysis of gene expression. Science 270:484-487.

13.Agaton, C., P. Unneberg, M. Sievertzon, A. Holmberg, M. Ehn, M. Larsson, J. Odeberg, M. Uhlen, and J. Lundeberg. 2002. Gene expression analysis by signature pyrosequencing. Gene 289:31-39.

14.DeRisi, J.L., V.R. Iyer, and P.O. Brown. 1997. Exploring the metabolic and genetic control of gene expression on a genome scale. Science 278:680-686.

15.Lashkari, D.A., J.L. DeRisi, J.H. McCusker, A.F. Namath, C. Gentile, S.Y. Hwang, P.O. Brown, and R.W. Davis. 1997. Yeast microarrays for genome wide parallel genetic and gene expression analysis. Proc. Natl. Acad. Sci. USA 94:13057-13062.

16.Lipshultz, R.J., S.P. Fodor, T.R. Gingeras, and D.J. Lockhart. 1999. High density synthetic oligonucleotide arrays. Nat. Genet. 21:20-24.

17.Lockhart, D.J. and E.A. Winzeler. 2000. Genomics, gene expression and DNA arrays. Nature 405:827-836.

18.Schena, M., D. Shalon, R.W. Davis, and P.O. Brown. 1995. Quantitative monitoring of gene expression patterns with a complementary DNA mi- 
croarray. Science 270:467-470.

19.Pollack, J.R., C.M. Perou, A.A. Alizadeh, M.B. Eisen, A. Pergamenschikov, and C.F. Williams. 1999. Genome-wide analysis of DNA copy number changes using cDNA microarrays. Nat. Genet. 23:41-46.

20.Frolov, A., A.H. Prowse, L. Vanderveer, B. Bove, H. Wu, and A.K. Godwin. 2002. DNA array-based method for detection of large rearrangements in the BRCA1 gene. Genes Chromosomes Cancer 35:232-241.

21.Haab, B.B. 2001. Advances in protein microarray technology for protein expression and interaction profiling. Curr. Opin. Drug Discov. Dev. 4:116123.

22.Fodor, S.P., J.L. Read, M.C. Pirrung, L. Stryer, A.T. Lu, and D. Solas. 1991. Light-directed, spatially addressable parallel chemical synthesis. Science 251:767-773.

23.Rose, S.D. 2002. Spotted arrays: technology overview, p. 3-14. In J.A. Warrington, C.R.Todd, and D. Wong (Eds.), Microarrays and Cancer Research. Eaton Publishing, Westboro, MA.

24. Okamoto, T., T. Suzuki, and N. Yamamoto. 2000. Microarray fabrication with covalent attachment of DNA using bubble jet technology. Nat. Biotechnol. 18:438-441.

25.Afshari, C.A., E.F. Nuwaysir, and J.C. Barrett. 1999. Application of complementary DNA microarray technology to carcinogen identification, toxicology, and drug safety evaluation. Cancer Res. 59:4759-4760.

26.Khan, J., R. Simon, M. Bittner, Y. Chen, S.B. Leighton, T. Pohida, P.D. Smith, Y. Jiang, et al. 1998. Gene expression profiling of alveolar rhabdomyosarcoma with cDNA microarrays. Cancer Res. 58:5009-5013.

27.Marx, J. 2000. Medicine. DNA arrays reveal cancer in its many forms. Science 289:1670-1672.

28.Bittner, M., P. Meltzer, Y. Chen, Y. Jiang, E. Seftor, M. Hendrix, M. Radmacher, R. Simon, et al. 2000. Molecular classification of cutaneous malignant melanoma by gene expression profiling. Nature 406:536-540.

29.DeRisi, J., L. Penland, P.O. Brown, M.L. Bittner, P.S. Meltzer, M. Ray, Y. Chen, Y.A. Su, and J.M. Trent. 1996. Use of a cDNA microarray to analyse gene expression patterns in human cancer. Nat. Genet. 14:457-460.

30.Pease, A.C., D. Solas, E.J. Sullivan, M.T. Cronin, C.P. Holmes, and S.P. Fodor. 1994. Light-generated oligonucleotide arrays for rapid DNA sequence analysis. Proc. Natl. Acad. Sci. USA 91:5022-5026.

31.Perou, C.M., S.S. Jeffrey, M. van de Rijn, C.A. Rees, M.B. Eisen, D.T Ross, A. Pergamenschikov, C.F. Williams, et al. 1999. Distinctive gene expression patterns in human mammary epithelial cells and breast cancers. Proc. Natl. Acad. Sci. USA 96:9212-9217.

32.Golub, T.R., D.K. Slonim, P. Tamayo, C. Huard, M. Gaasenbeek, J.P. Mesirov, H. Coller, M.L. Loh, et al. 1999. Molecular classification of cancer: class discovery and class prediction by gene expression monitoring. Science 286:531-537.

33.Yeoh, E.J., M.E. Ross, S.A. Shurtleff, W.K. Williams, D. Patel, R. Mahfouz, F.G. Behm, S.C. Raimondi, et al. 2002. Classification, subtype discovery, and prediction of outcome in pediatric acute lymphoblastic leukemia by gene expression profiling. Cancer Cell 1:133-143.

34. Ono, K., T. Tanaka, T. Tsunoda, O. Kitahara, C. Kihara, A. Okamoto, K. Ochiai, T. Takagi, and Y. Nakamura. 2000. Identification by cDNA microarray of genes involved in ovarian carcinogenesis. Cancer Res. 60:50075011

35.Schwartz, D.R., S.L. Kardia, K.A. Shedden, R. Kuick, G. Michailidis, J.M. Taylor, D.E. Misek, R. Wu, et al. 2002. Gene expression in ovarian cancer reflects both morphology and biological behavior, distinguishing clear cell from other poor-prognosis ovarian carcinomas. Cancer Res. 62:4722-4729.

36.Perou, C.M., T. Sorlie, M.B. Eisen, M. van de Rijn, S.S. Jeffrey, C.A. Rees, J.R. Pollack, D.T. Ross, et al. 2000. Molecular portraits of human breast tumours. Nature 406:747-752.

37.Sorlie, T., C.M. Perou, R. Tibshirani, T. Aas, S. Geisler, H. Johnsen, T. Hastie, M.B. Eisen, et al. 2001. Gene expression patterns of breast carcinomas distinguish tumor subclasses with clinical implications. Proc. Natl. Acad. Sci. USA 98:10869-10874.

38.Celis, J.E., M. Kruhoffer, I. Gromova, C. Federiksen, M. Ostergaard, T. Thykjaer, P. Gromov, J. Yu, et al. 2000. Gene expression profiling: monitoring transcription and translation products using DNA microarrays and protoemics. FEBS Lett. 480:2-16.

39.Bhattacharjee, A., W.G. Richards, J. Staunton, C. Li, S. Monti, P. Vasa, C. Ladd, J. Beheshti, et al. 2001. Classification of human lung carcinomas by mRNA expression profiling reveals distinct adenocarcinoma subclasses. Proc Natl. Acad. Sci. USA 98:13790-13795.

40.Garber, M.E., O.G. Troyanskaya, K. Schluens, S. Petersen, Z. Thaesler, M.
Pacyna-Gengelbach, M. van de Rijn, G.D. Rosen, et al. 2001. Diversity of gene expression in adenocarcinoma of the lung. Proc. Natl. Acad. Sci. USA 98:13784-13789.

41.Dhanasekaran, S.M., T.R. Barrette, D. Ghosh, R. Shah, S. Varambally, K. Kurachi, K.J. Pienta, M.A. Rubin, and A.M. Chinnaiyan. 2001. Delineation of prognostic biomarkers in prostate cancer. Nature 412:822-826.

42.Ismail, R.S., R.L. Baldwin, J. Fang, D. Browning, B.Y. Karlan, J.C. Gasson, and D.D. Chang. 2000. Differential gene expression between normal and tumor-derived ovarian epithelial cells. Cancer Res. 60:6744-6749.

43.Mok, S.C., J. Chao, S. Skates, K. Wong, G.K. Yiu, M.G. Muto, R.S Berkowitz, and D.W. Cramer. 2001. Prostasin, a potential serum marker for ovarian cancer: identification through microarray technology. J. Natl. Cancer Inst. 93:1458-1464.

44.Sawiris, G.P., C.A. Sherman-Baust, K.G. Becker, C. Cheadle, D. Teichberg, and P.J. Morin. 2002. Development of a highly specialized cDNA array for the study and diagnosis of epithelial ovarian cancer. Cancer Res. 62:2923-2928.

45.Shridhar, V., J. Lee, A. Pandita, S. Iturria, R. Avula, J. Staub, M. Morrissey, E. Calhoun, et al. 2001. Genetic analysis of early- versus late-stage ovarian tumors. Cancer Res. 61:5895-5904.

46.Shridhar, V., A. Sen, J. Chien, J. Staub, R. Avula, S. Kovats, J. Lee, J. Lillie, and D.I. Smith. 2002. Identification of underexpressed genes in early- and late-stage primary ovarian tumors by suppression subtraction hybridization. Cancer Res. 62:262-270.

47.Welsh, J.B., P.P. Zarrinkar, L.M. Sapinoso, S.G. Kern, C.A. Behling, B.J. Monk, D.J. Lockhart, R.A. Burger, and G.M. Hampton. 2001. Analysis of gene expression profiles in normal and neoplastic ovarian tissue samples identifies candidate molecular markers of epithelial ovarian cancer. Proc. Natl. Acad. Sci. USA 98:1176-1181.

48.Wong, K.-K., R.S. Cheng, R.S. Berkowitz, and S.C. Mok. 2002. Gene expression analysis of ovarian cancer cells by cNDA microarrays, p. 127-138. In J.A. Warrington, C.R. Todd, and D. Wong (Eds.), Microarrays and Can cer Research. Eaton Publishing, Westborough, MA.

49.Alevizos, I., M. Mahadevappa, X. Zhang, H. Ohyama, Y. Kohno, M. Posner, G.T. Gallagher, M. Varvares, et al. 2001. Oral cancer in vivo gene expression profiling assisted by laser capture microdissection and microarray analysis. Oncogene 20:6196-6204.

50.Todd, R., J.S. Gutkind, E.J. Shillitoe, and D. Wong. 2002. Solid tumors: microarray analysis of oral cancers, p. 139-153. In J.A. Warrington, C.R Todd, and D. Wong (Eds.), Microarrays and Cancer Research. Eaton Publishing, Westborough, MA

51.Du, F., M. Mahadevappa, J.A. Warrington, and A.M. Bowcock. 2002 Gene expression changes in endometrial cancer, p. 113-125. In J.A. Warrington, C.R. Todd, and D. Wong, (Eds.), Microarrays and Cancer Research. Eaton Publishing, Westborough, MA.

52.Clark, E.A., T.R. Golub, E.S. Lander, and R.O. Hynes. 2000. Genomic analysis of metastasis reveals an essential role for RhoC. Nature 406:532-535.

53.Hegde, P., R. Qi, R. Gaspard, K. Abernathy, S. Dharap, J. Earle-Hughes, C. Gay, N.U. Nwokekeh, et al. 2001. Identification of tumor markers in models of human colorectal cancer using a 19,200-element complementary DNA microarray. Cancer Res. 61:7792-7797.

54.Notterman, D.A., C.J. Shawber, and A.J. Levine. 2002. Tumor biology and microarray analysis of solid tumors: colorectal cancer as a model system, p. 81-111. In J.A. Warrington, C.R. Todd, and D. Wong (Eds.), Microarrays and Cancer Research. Eaton Publishing, Westborough, MA.

55.Paweletz, C.P. and L.A. Liotta. 2002. Tumor classification: gene analysis using DNA microarrays, p. 61-77. In J.A. Warrington, C.R. Todd, and D Wong (Eds.), Microarrays and Cancer Research. Eaton Publishing, Westborough, MA.

56.Bayani, J., J.D. Brenton, P.F. Macgregor, B. Beheshti, M. Albert, D. Nallainathan, J. Karaskova, B. Rosen, et al. 2002. Parallel analysis of sporadic primary ovarian carcinomas by spectral karyotyping, comparative genomic hybridization, and expression microarrays. Cancer Res. 62:3466-3476.

57.Barlund, M., F. Forozan, J. Kononen, L. Bubendorf, Y. Chen, M.L. Bittner, J. Torhorst, P. Haas, et al. 2000. Detecting activation of ribosomal protein S6 kinase by complementary DNA and tissue microarray analysis. J. Natl. Cancer Inst. 92:1252-1259.

58.Monni, O., M. Barlund, S. Mousses, J. Kononen, G. Sauter, M. Heiskanen, P. Paavola, K. Avela, et al. 2001. Comprehensive copy number and gene expression profiling of the 17q23 amplicon in human breast cancer Proc. Natl. Acad. Sci. USA 98:5711-5716.

59.Carson, D.A. and A. Lois. 1995. Cancer progression and p53. Lancet 
346:1009-1011.

60.Fisher, D.E. 2001. The p53 tumor suppressor: critical regulator of life and death in cancer. Apoptosis 6:7-15.

61.Harris, C.C. 1996. Structure and function of the p53 tumor suppressor gene: clues for rational cancer therapeutic strategies. J. Natl. Cancer Inst. $88: 1442-1455$

62.Hirota, S. 2001. Gastrointestinal stromal tumors: their origin and cause. Int. J. Clin. Oncol. 6:1-5.

63.Hirota, S., K. Isozaki, Y. Moriyama, K. Hashimoto, T. Nishida, S. Ishiguro, K. Kawano, M. Hanada, et al. 1998. Gain-of-function mutations of ckit in human gastrointestinal stromal tumors. Science 279:577-580.

64.Pelengaris, S., M. Khan, and G. Evan. 2002. c-MYC: more than just a matter of life and death. Nat. Rev. Cancer 2:764-776.

65.Knudson, A.G. 1971. Mutation and cancer: statistical study of retinoblastoma. Proc. Natl. Acad. Sci. USA 68:820-823.

66.Grady, W.M. and S.D. Markowitz. 2002. Genetic and epigenetic alterations in colon cancer. Annu. Rev. Genomics Hum. Genet. 3:101-128.

67.Alizadeh, A.A., M.B. Eisen, R.E. Davis, C. Ma, I.S. Lossos, A. Rosenwald, J.C. Boldrick, H. Sabet, et al. 2000. Distinct types of diffuse large B-cell lymphoma identified by gene expression profiling. Nature 403:503-511.

68.Ross, D.T., U. Scherf, M.B. Eisen, C.M. Perou, C. Rees, P. Spellman, V. Iyer, S.S. Jeffrey, et al. 2000. Systematic variation in gene expression patterns in human cancer cell lines. Nat. Genet. 24:227-235.

69.Eisen, M.B., P.T. Spellman, P.O. Brown, and D. Botstein. 1998. Cluster analysis and display of genome-wide expression patterns. Proc. Natl. Acad. Sci. USA 95:14863-14868.

70.Getz, G., E. Levine, and E. Domany. 2000. Coupled two-way clustering analysis of gene microarray data. Proc. Natl. Acad. Sci. USA 97:1207912084.

71.Lukashin, A.V. and R. Fuchs. 2001. Analysis of temporal gene expression profiles: clustering by simulated annealing and determining the optimal number of clusters. Bioinformatics 17:405-414.

72.Kerr, M.K. and G.A. Churchill. 2001. Bootstrapping cluster analysis: assessing the reliability of conclusions from microarray experiments. Proc. Natl. Acad. Sci. USA 98:8961-8965.

73.Yeung, K.Y., D.R. Haynor, and W.L. Ruzzo. 2001. Validating clustering for gene expression data. Bioinformatics 17:309-318.

74.Heyer, L.J., S. Kruglyak, and S. Yooseph. 1999. Exploring expression data: identification and analysis of coexpressed genes. Genome Res. 9:1106-1115.

75.Alter, O., P.O. Brown, and D. Botstein. 2000. Singular value decomposition for genome-wide expression data processing and modeling. Proc. Natl. Acad. Sci. USA 97:10101-10106.

76.Futschik, M.E. and N.K. Kasabov. 2002. Fuzzy clustering of gene expression data, p. 414-419. 2002 IEEE International Conference on Fuzzy Systems. IEEE, Honolulu, HI.

77.Gasch, A.P. and M.B. Eisen. 2002. Exploring the conditional coregulation of yeast gene expression through fuzzy k-means clustering. Genome Biol. 3:RESEARCH0059.

78.Tamayo, P., D. Slonim, J. Mesirov, Q. Zhu, S. Kitareewan, E. Dmitrovsky, E.S. Lander, and T.R. Golub. 1999. Interpreting patterns of gene expression with self-organizing maps: methods and application to hematopoietic differentiation. Proc. Natl. Acad. Sci. USA 96:2907-2912.

79.Khan, J., J.S. Wei, M. Ringner, L.H. Saal, M. Ladanyi, F. Westermann, F. Berthold, M. Schwab, et al. 2001. Classification and diagnostic prediction of cancers using gene expression profiling and artificial neural networks. Nat. Med. 7:673-679.

80.Brown, M.P., W.N. Grundy, D. Lin, N. Cristianini, C.W. Sugnet, T.S. Furey, M. Ares, Jr., and D. Haussler. 2000. Knowledge-based analysis of microarray gene expression data by using support vector machines. Proc. Natl. Acad. Sci. USA 97:262-267.

81.Jajuga, K., A. Sokolowski, and H.-H. Bock. 2002. Classification, Clustering, and Data Analysis. Springer-Verlag, New York.

82.Kaufman, L. and P.J. Rousseeuw. 1990. Finding Groups in Data: An Introduction to Cluster Analysis. Wiley, New York.

83.Soukas, A., P. Cohen, N.D. Socci, and J.M. Friedman. 2000. Leptin-specific patterns of gene expression in white adipose tissue. Genes Dev. 14:963980 .

84.Soukas, A., N.D. Socci, B.D. Saatkamp, S. Novelli, and J.M. Friedman. 2001. Distinct transcriptional profiles of adipogenesis in vivo and in vitro. J. Biol. Chem. 276:34167-34174.

85.Geman, S. and D. Geman. 1984. Stochastic relaxation, Gibbs distributions, and the Bayesian restoration of images. IEEE Transactions on Pattern Analy- sis and Machine Intelligence PAMI-6:721-741

86.Kerr, M.K., C.A. Afshari, L. Bennett, P. Bushel, J. Martinez, N.J. Walker, and G.A. Churchill. 2002. Statistical analysis of a gene expression microarray experiment with replication. Statistica Sinica 12:203-218.

87.Moloshok, T.D., R.R. Klevecz, J.D. Grant, F.J. Manion, W.I. Speier, and M.F. Ochs. 2002. Application of Bayesian decomposition for analysing microarray data. Bioinformatics 18:566-575.

88.Parmigiani, G., E. Garrett, R. Anbazhagan, and E. Gabrielson. 2002. A statistical framework for expression-based molecular classification in cancer. J. R. Statistical Soc. [B] 64:717-736.

89.Gilman, A. and A.P. Arkin. 2002. Genetic "code": representations and dynamical models of genetic components and networks. Annu. Rev. Genomics Hum. Genet. 3:341-369.

90.Kaufman, S. 1993. The Origins of Order: Self-Organizatin and Selection in Evolution. Oxford University Press, Oxford.

91.Hartemink, A.J., D.K. Gifford, T.S. Jaakkola, and R.A. Young. 2001. Using graphical models and genomic expression data to statistically validate models of genetic regulatory networks. Pac. Symp. Biocomput. 422-433.

92. Tanaka, H., H. Arakawa, T. Yamaguchi, K. Shiraishi, S. Fukuda, K. Matsui, Y. Takei, and Y. Nakamura. 2000. A ribonucleotide reductase gene involved in a p53-dependent cell-cycle checkpoint for DNA damage. Nature 404:42-49.

93.Yu, J., L. Zhang, P.M. Hwang, C. Rago, K.W. Kinzler, and B. Vogelstein. 1999. Identification and classification of p53-regulated genes. Proc. Natl. Acad. Sci. USA 96:14517-14522.

94.Harkin, D.P. 2000. Uncovering functionally relevant signaling pathways using microarray- based expression profiling. Oncologist 5:501-507.

95.Sibisi, S. and J. Skilling. 1997. Prior distributions on measure space. J. Royal Statistical Soc. [B] 59:217-235.

96.Cho, R.J., M.J. Campbell, E.A. Winzeler, L. Steinmetz, A. Conway, L. Wodicka, T.G. Wolfsberg, A.E. Gabrielian, et al. 1998. A genome-wide transcriptional analysis of the mitotic cell cycle. Mol. Cell 2:65-73.

97.Spellman, P.T., G. Sherlock, M.Q. Zhang, V.R. Iyer, K. Anders, M.B. Eisen, P.O. Brown, D. Botstein, and B. Futcher. 1998. Comprehensive identification of cell cycle-regulated genes of the yeast Saccharomyces cerevisiae by microarray hybridization. Mol. Biol. Cell 9:3273-3297.

98.Bidaut, G., T.D. Moloshok, J.D. Grant, F.J. Manion, and M.F. Ochs. 2002. Bayesian Decomposition analysis of gene expression in yeast deletion mutants, p. 105-122. In K. Johnson and S. Lin (Eds.), Methods of Microarray Data Analysis II. Kluwer Academic, Boston.

99.Hughes, T.R., M.J. Marton, A.R. Jones, C.J. Roberts, R. Stoughton, C.D. Armour, H.A. Bennett, E. Coffey, et al. 2000. Functional discovery via a compendium of expression profiles. Cell 102:109-126.

100.Moloshok, T.D., D. Datta, A.V. Kossenkov, and M.F. Ochs. Bayesian decomposition classification of the project normal data set. In K. Johnson and S. Lin (Eds.), Methods of Microarray Data Analysis III. Kluwer Academic, Boston. (In press.)

101.Pritchard, C.C., L. Hsu, J. Delrow, and P.S. Nelson. 2001. Project normal: defining normal variance in mouse gene expression. Proc. Natl. Acad. Sci. USA 98:13266-13271.

\section{Address correspondence to:}

Dr. Michael F. Ochs

Fox Chase Cancer Center

7701 Burholme Avenue

Philadelphia, PA 19111, USA

e-mail:m_ochs@fccc.edu

Dr. Andrew K. Godwin

Fox Chase Cancer Center

7701 Burholme Avenue

Philadelphia, PA 19111, USA

e-mail:a_godwin@fccc.edu 